\title{
Low-resolution Structure of Recombinant Human Granulocyte-Macrophage Colony Stimulating Factor
}

\author{
Kay Diederichs ${ }^{1}$, Steven Jacques ${ }^{1}$, Tom Boone ${ }^{2}$ and P. Andrew Karplus ${ }^{1} \dagger$ \\ ${ }^{1}$ Section of Biochemistry, Molecular and Cell Biology \\ Cornell University, Ithaca, NY 14853, U.S.A. \\ ${ }^{2}$ AMGEN, 1840 Dehavilland Drive, Thousand Oaks, CA 91320-1789, U.S.A.
}

\begin{abstract}
A recombinant form of human granulocyte-macrophage colony stimulating factor (GM-CSF) which contains no carbohydrate has been crystallized. Multiple isomorphous replacement analysis using five heavy-atom derivatives has yielded an image of the structure at $6 \AA$ resolution that showed two molecules per asymmetric unit and allowed determination of the non-erystallographic symmetry transformation. The $6 \AA$ resolution result shows that the core of GM-CSF consists of four helices. The angles at which the helices pack together distinguishes this structure from known antiparallel four-helix bundle proteins. Consideration of the amino acid sequence properties and previous structural characterizations of GM-CSF leads to an assignment of the probable protein segments that form the helices.
\end{abstract}

Keywords: granulocyte--macrophage colony stimulating factor; lymphokine; crystallization; X-ray diffraction; protein structure

\begin{abstract}
Granulocyte-macrophage colony stimulating factor (GM-CSF $\ddagger$ ) is a member of the family of protein factors called lymphokines that regulate the proliferation, differentiation and activation of cells in the hematopoietic lineage (Clark \& Kamen, 1987; Metcalf, 1989). GM-CSF is produced in vivo by macrophages, T-cells, fibroblasts and endothelial cells. As its name suggests, GM-CSF stimulates granulocyte and macrophage progenitor cells during normal hematopoiesis. It also has been shown to play a more direct role in host defense by stimulating the activities of mature granulocytes and macrophages, such as phagocytosis, superoxide production and cell mediated cytotoxicity. These activities make GM-CSF an attractive therapeutic agent, and it has proven effective in clinical settings for strengthening the cellular immune response (Groopman et al., 1987; Grossberg et al., 1989) and stimulating hematopoietic reconstitution (Brandt et al., 1988; Antman et al., 1988). It is clear that most, if not all activities of GM-CSF are initiated by the
\end{abstract}

$\dagger$ Author to whom all correspondence should be addressed.

†Abbreviations used: GM-CSF, granulocytemacrophage colony stimulating factor; PEG, polyethylene glycol; $p$ CMBS, $p$-chloromercuribenzenesulfonic acid; m.i.r., multiple isomorphous replacement; r.m.s., root-mean-square. specific binding of GM-CSF to either a high-affinity receptor with $K_{\mathrm{d}} \approx 40$ pM (Dipersio et al., 1988) or a lower-affinity receptor with $K_{\mathrm{d}} \approx 1 \mathrm{nM}$ (Chiba et al., 1990).

We have initiated structural studies on human GM-CSF in order to elucidate accurately the structural features required for its action. Two methods for crystallizing GM-CSF have been published (LaLonde et al., 1989; Reichert et al., 1990). Here we present a third set of conditions for crystallization and an image of the structure at $6 \AA$ resolution (1 $\AA=0.1 \mathrm{~nm}$ ). In contrast with circular dichroism studies that indicated that GM-CSF has similar amounts of $\alpha$-and $\beta$-structure (Wingfield et al., 1988 ), we find that the protein is predominantly $\alpha$-helical, which allows useful information to be gleaned from the $6 \AA$ resolution structure.

Recombinant human GM-CSF was purified from Escherichia coli (Boone et al., 1987; Burgess et al., 1987 ) and stored at $10 \mathrm{mg} / \mathrm{ml}$ in $2 \mathrm{~mm}$-potassium phosphate ( $\mathrm{pH} 7$ ). The recombinant protein is 127 residues long, has the native disulfide groups (54 to 96 and 88 to 121; Schrimsher et al., 1987), and its sequence matches that reported by Miyatake et al. (1985) for a genomic clone. Although it lacks glycosylation, it is about 20 -fold more active than the natural glycosylated form (Kaushansky et al., 1987; Cebon et al., 1990). Crystals were obtained by the hanging drop method between $\mathrm{pH} 5.5$ and 6.5 at 
Table 1

Data collection and phasing statistics

\begin{tabular}{|c|c|c|c|c|c|c|}
\hline \multirow[b]{2}{*}{ Data set ${ }^{\dagger}$} & \multirow{2}{*}{$\begin{array}{l}\text { Soak concentration } \\
(\mathrm{mm})\end{array}$} & \multirow[b]{2}{*}{ Time (days) } & \multirow[b]{2}{*}{$R_{\text {sym }}(\%)_{+}^{\ddagger}$} & \multirow[b]{2}{*}{$R_{\text {native }}(\%) \S$} & \multicolumn{2}{|c|}{ Phasing power\| } \\
\hline & & & & & $\infty-6 \AA$ & $6-3 \AA$ \\
\hline Native (phos) & & & $5 \cdot 8$ & & & \\
\hline$p$ CMBS & 5 & 1.5 & $6 \cdot 0$ & $9 \cdot 5$ & 10 & $0 \cdot 6$ \\
\hline $\mathrm{NaAuCl}_{4}$ & $0 \cdot 2$ & 1 & $6 \cdot 0$ & $8 \cdot 8$ & $1 \cdot 0$ & 0.5 \\
\hline $\mathrm{Pt}\left(\mathrm{EtNH}_{2}\right)_{2} \mathrm{Cl}_{2}$ & 5 & 2 & 6.7 & $17 \cdot 9$ & $1 \cdot 3$ & 0.8 \\
\hline Native (cit) & & & $6 \cdot 3$ & $\ldots$ & & \\
\hline $\mathrm{EtHgCl}$ & 1 & $0 \cdot 7$ & $5 \cdot 4$ & 8.8 & $1 \cdot 2$ & 0.5 \\
\hline $\mathrm{HgNO}_{3}$ & $0 \cdot 2$ & 11 & $5 \cdot 0$ & 844 & $1 \cdot 0$ & $0 \cdot 6$ \\
\hline
\end{tabular}

All data sets were $99 \%$ complete to $3 \AA$ resolution and had approximately 3 -fold redundancy. X-ray data were collected using a single San Diego Multiwire Systems detector on a Rigaku RU-200 rotating anode generator. Data collection and data reduction protocols recommended for this detector were used (Hamlin 1985; Howard et al. 1985; Xuong et al. 1985). Typically. data between 10 A and $2.8 \AA$ resolution were collected by $4 \omega$-sweeps of $70^{\circ}$ with a detector at $\theta_{c}=20^{\circ}$, and then data between $\infty$ and $4 \AA$ resolution were collected by $3 \omega$-sweeps of $70^{\circ}$ with the detector at $\theta_{\mathrm{c}}=10^{\circ}$. The exposure times were $20 \mathrm{~s}$ for each $0 \cdot 1^{\circ}$ frame in $\omega$ and the $\left(\mathrm{rr}^{\circ} \mathrm{stal}\right.$ to detector distance was $780 \mathrm{~mm}$. The data at $3 \AA$ resolution decrease about $35 \%$ during the $20 \mathrm{~h}$ data collection.

$\dagger$ Data sets are as follows: native (phos), native crystal grown in citrate/phosphate buffer; $p$ CMBS, $p$-chloromercuribenzenesulfoni: acid; $\mathrm{Pt}\left(\mathrm{EtNH}_{2}\right)_{2} \mathrm{Cl}_{2}$, diethylamino platinum dichloride; native (eit), native crystal grown in citrate; EtHgCl, ethyl mercuric chloride.

$\ddagger R_{\text {sym }}=\sum_{h k l}|I-\langle I\rangle| / \sum_{h k l} I$, where $\langle I\rangle$ is the average intensity of symmetry-related reflections.

$\S R_{\text {native }}=\sum_{h k l}\left|F_{2}-F_{1}\right| / \sum_{h k l} F_{1}$, where $F_{1}$ is the structure factor from the appropriate native crystal and $F_{2}$ is that of the derivative.

II Refinement of the heavy-atom models was carried out by the method of Jickerson et al. (1968). Phasing power is the r.m.s. heavyatom signal divided by the r.m.s. lack-of-closure error.

$4^{\circ} \mathrm{C}$. For optimal growth, the reservoir contained $21 \%$ (w/v) PEG-8000 in $0.2 \mathrm{~m}$-sodium citrate/phos-

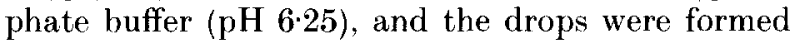
by mixing equal volumes of the protein and reservoir solutions. Sometimes $0.2 \mathrm{~m}$-trisodium citrate $(\mathrm{pH} \mathrm{6.25)}$ was used in place of the citrate/phosphate buffer in order to allow the use of heavy-atom compounds that precipitate in phosphate buffer. The crystals grow within 1 to 2 weeks to sizes of up to $0.8 \mathrm{~mm} \times 0.4 \mathrm{~mm} \times 0.2 \mathrm{~mm}$. They show $P 2_{1} 2_{1} 2_{1}$ symmetry with $a=47 \cdot 6 \AA, b=59 \cdot 1 \AA, c=126 \cdot 7 \AA$ and apparently correspond to the crystals obtained by both LaLonde et al. (1989) and Reichert et al.
(1990). A fresh native crystal diffracts to $2 \cdot 2 \mathrm{~A}$ resolution, but the highest resolution data deteriorate rapidly during irradiation.

Reichert et al. (1990) used PEG-8000 for crystallization but they obtained crystals at higher $\mathrm{pH}$ values between 7 and 8 . LaLonde et al. (1989) crystallized GM-CSF from high salt solutions, also near pH 8. Apparently, the packing arrangement adopted by the GM-CSF molecules in this crystal form is fairly dominant, as the same interactions are stabilized at $\mathrm{pH}$ values near 6 and 8 using either salt or PEG as precipitant. The unit cell volume suggests that the asymmetric unit contains either

Table 2

Heavy-atom derivative models

\begin{tabular}{|c|c|c|c|c|c|c|}
\hline \multirow[b]{2}{*}{ Derivative } & \multirow[b]{2}{*}{ Site } & \multicolumn{3}{|c|}{ Fractional co-ordinates } & \multirow{2}{*}{$\begin{array}{c}\text { Relative } \\
\text { occupaney }\end{array}$} & \multirow[b]{2}{*}{$B\left(\AA^{2}\right)$} \\
\hline & & $x$ & $y$ & $z$ & & \\
\hline \multirow[t]{2}{*}{$p$ CMBS } & 1 & $0 \cdot 093$ & $0 \cdot 094$ & 0.191 & 54 & 37 \\
\hline & 2 & $0 \cdot 592$ & 0.937 & $0 \cdot 203$ & 65 & 212 \\
\hline \multirow[t]{4}{*}{$\mathrm{NaAuCl}_{4}$} & 1 & $0 \cdot 155$ & 0.069 & $0 \cdot 181$ & 24 & 3 \\
\hline & 2 & 0.545 & 0.957 & $0 \cdot 217$ & 21 & 28 \\
\hline & 3 & $0 \cdot 244$ & 0.987 & $0 \cdot 201$ & 17 & 5 \\
\hline & 4 & $0 \cdot 376$ & 0.945 & $0 \cdot 210$ & 17 & 46 \\
\hline \multirow{4}{*}{$\mathrm{Pt}\left(\mathrm{EtNH}_{2}\right)_{2} \mathrm{Cl}_{2}$} & 1 & $0 \cdot 661$ & 0.077 & $0 \cdot 252$ & 207 & 195 \\
\hline & 2 & $0 \cdot 134$ & $0 \cdot 237$ & $0 \cdot 154$ & 132 & 49 \\
\hline & 3 & 0.828 & 0.505 & $0 \cdot 145$ & 140 & 162 \\
\hline & 4 & $0 \cdot 469$ & 0.982 & $0 \cdot 224$ & 76 & 193 \\
\hline \multirow[t]{4}{*}{ EtHgCl } & 1 & 0.077 & $0 \cdot 098$ & $0 \cdot 195$ & 48 & 52 \\
\hline & 2 & 0.564 & 0.956 & 0.211 & 67 & 134 \\
\hline & 3 & $0 \cdot 249$ & 0.992 & $0 \cdot 201$ & 39 & 17 \\
\hline & 4 & $0 \cdot 380$ & $0 \cdot 945$ & 0.209 & 28 & 26 \\
\hline \multirow[t]{4}{*}{$\mathrm{HgNO}_{3}$} & 1 & $0 \cdot 167$ & 0.068 & $0 \cdot 181$ & 16 & 22 \\
\hline & 2 & 0.555 & 0.960 & $0 \cdot 215$ & 25 & 26 \\
\hline & 3 & $0 \cdot 248$ & 0.992 & $0 \cdot 201$ & 36 & 11 \\
\hline & 4 & $0 \cdot 387$ & 0.942 & 0.209 & 27 & 26 \\
\hline
\end{tabular}


two or three molecules, but self-rotation functions did not yield a clear solution (see also Reichert et al., 1990).

We have approached the structure solution by multiple isomorphous replacement methods. Heavyatom soaks of up to 48 hours were carried out in a crystal storage buffer containing $24 \%(\mathrm{w} / \mathrm{v})$ PEG-8000. However, the crystals degrade slowly in the storage buffer, so longer soaks were carried out by adding the heavy-atom solution directly to the hanging drops.

Five heavy-atom compounds have resulted in derivatives that could be used for phasing (Table 1). The $p$ CMBS derivative was the first to be interpreted on the basis of difference Patterson synthesis, and the remaining were interpreted by the combined use of difference Fouriers and difference Pattersons. Three derivatives were found using the original citrate/phosphate buffer system. Because the salts of many heavy-atoms have low solubility in phosphate buffer, we began to produce crystals in citrate buffer, so that a wider variety of salts could be screened. Two further derivatives, ethylmercuric chloride and mercuric nitrate, were found, although their binding sites were similar to each other and to the $\mathrm{NaAuCl}_{4}$ derivative (Table 2). A native data set collected for citrate-buffered crystals showed an $R$-factor of $9 \cdot 7 \%$ versus the citrate/phosphate buffered crystals, suggesting that small systematic differences exist between the crystals. Phase determination and refinement programs were modified to allow for two native data sets, and phase circles for each derivative data set were generated with reference to the appropriate native data. The final heavy-atom models are summarized in Table 2. Among the 18 sites seen in the five derivatives, only eight fundamentally unique binding sites $(>4 \AA$ apart) are represented. All the derivatives except platinum show low $R$-factors versus the native data (Table 1) as is consistent with the low occupancies seen during refinement (Table 2). We have tried to increase the signal by carrying out soaks at higher concentrations or for longer times, but in all cases the diffraction by the crystals was greatly impaired or the crystals dissolved in the heavy-atom solution.

The overall figure of merit at $6 \AA$ resolution was $0 \cdot 8$. The initial electron density map calculated at $6 \AA$ resolution showed very good molecule/solvent contrast and two molecules per asymmetric unit (Fig. 1). Approximate centers of mass for the two molecules were obtained by inspection and a noncrystallographic symmetry transformation, which involves a $42^{\circ}$ rotation, was determined that maximized the correlation of the electron densities from the two molecules (see the legend to Fig. 1). The electron density correlation at $6 \AA$ resolution is as high as 0.78 , suggesting that the main features of the molecule visible at $6 \AA$ resolution are conserved between the two molecules. It seemed that, with this high a correlation, the non-crystallographic symmetry should have been visible in the selfrotation function that was originally sampled by Eulerian angles. When the self-rotation function

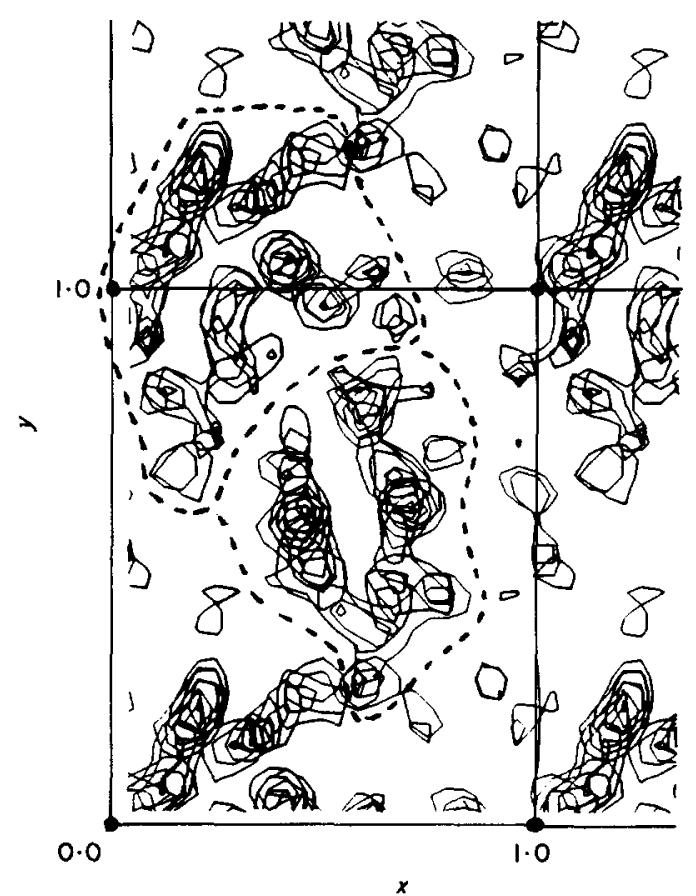

Figure 1. The $6 \AA$ resolution m.i.r. electron density map. More than a full unit cell in $x$ and $y$ are shown for a $12 \AA$ thick slab along the $z$-direction. Contours are at 20 , 40,60 and $80 \%$ of the maximum (r.m.s. level $=17 \%$ ). The molecule/solvent contrast and the 2 molecules/asymmetric unit (enclosed by broken lines) are clearly seen. Exact values for translational and rotational parameters were obtained in the following way. As a lst step, approximate translation parameters were obtained from the electron density map, and the program X-PLOR (Brünger et al., 1987) was used to generate a list of the 50 highest peaks of the self-rotation function (resolution 15 to $4 \AA$, Patterson radius 5 to $20 \AA$ ), including the origin peak with Eulerian angles $(0,0,0)$. As a 2nd step, each possible rotation angle was refined using the real-space refinement program NCSREF (K. Diederichs, unpublished results), which iteratively adjusts the rotational and translational parameters to obtain an optimal superposition of the electron densities within a radius of $15 \AA$ of the 2 centers of mass. The result was unambiguous: the Eulerian angles corresponding to the origin peak refined to $(47,-8,-6)$ to yield a correlation coefficient between the electron densities of the molecules of $0 \cdot 78$. All other rotation angles gave a density correlation between 0.0 and $0 \cdot 30$. The translational parameters shifted by $6 \AA$ during the minimization to give final molecular "centers" of $(0 \cdot 312,0 \cdot 100,0 \cdot 070)$ and $(0 \cdot 607,0 \cdot 550,0 \cdot 172)$ in fractional co-ordinates.

was recalculated using polar angles (Rossmann \& Blow, 1962), the true solution is indeed the highest non-origin peak, and is well resolved from the origin (Fig. 2). This shows that polar angles and Eulerian angles, which sample rotation space differently, can be used complementarily for determining non-crystallographic symmetry relations.

Using the symmetry information, the phases were improved by solvent flattening (Wang, 1985; using a solvent content of $60 \%$ ) and non-crystallographic symmetry averaging of electron density maps at 


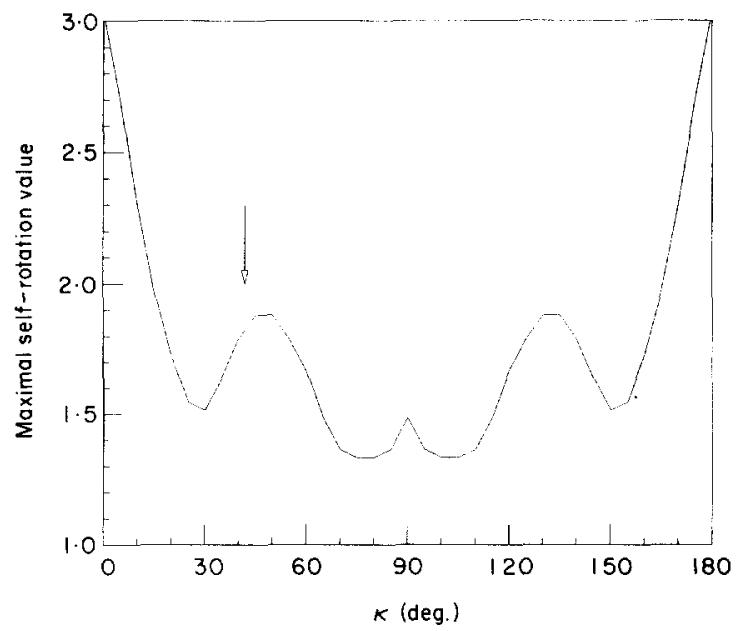

(a)

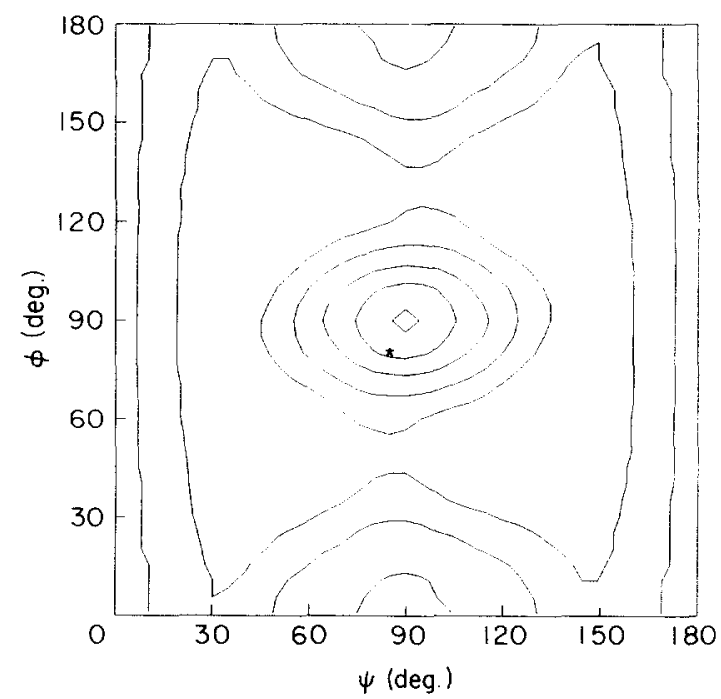

(b)

Figure 2. Non-crystallographic symmetry seen in the self-rotation function. (a) For each value of the rotation angle $\kappa$ (sampled every $\left.5^{\circ}\right)$, the rotation function was calculated with all possible rotation axes $(0 \leq \psi \leq 180$ and $0 \leq \phi \leq 180$. both in $5^{\circ}$ increments) and the maximal value obtained was entered in the plot. Although the unique information is obtained for $\kappa \leq 90, \kappa$ values are shown through $180^{\circ}$. The largest non-origin maximum occurs at $\kappa=45^{\circ}$ in agreement with the refined value (see arrow). (b) A contour map of the rotation function value for $\kappa=-42$ as a function of the rotation axis. Contours at $49,60,72,83$ and $95 \%$ of the maximum value for this section. The clear maximum at $\psi=90$, $\phi=90$ is consistent with the refined values of $\psi=85, \phi=80$ (shown by the asterisk, ${ }^{*}$ ). The plot has a center of symmetry at $\psi=90, \phi=90$.

both $6 \AA$ and $3 \AA$ resolution. Figure 3 shows the averaged electron density at $6 \AA$ resolution. The main features are four tubes of density, which are characteristic of $\alpha$-helices. All correspond in length to helices that contain roughly 14 to 18 residues. One of the helix-helix pairs is nearly parallel and the others cross each other at angles close to $45^{\circ}$ (Fig. 3). Although we do not have experimental proof for the proper handedness of this structure (i.e. whether the electron density shown in Fig. 3 corresponds to the true structure or its mirror image), we have chosen the hand shown by referring to studies (Chothia et al., 1981) that showed that, while interhelix packing angles near $-45^{\circ}$ were a very commonly observed arrangement, angles near $+45^{\circ}$ were observed rarely. This choice is in conflict with the handedness suggested by phasing statistics using the observed anomalous scattering signal (data not shown), and the question remains unresolved. Although we have calculated electron density maps at $3 \AA$ resolution, they are so noisy that we cannot assign the handedness, the directions or the connectivities of the individual helices. For the electron density calculated with data
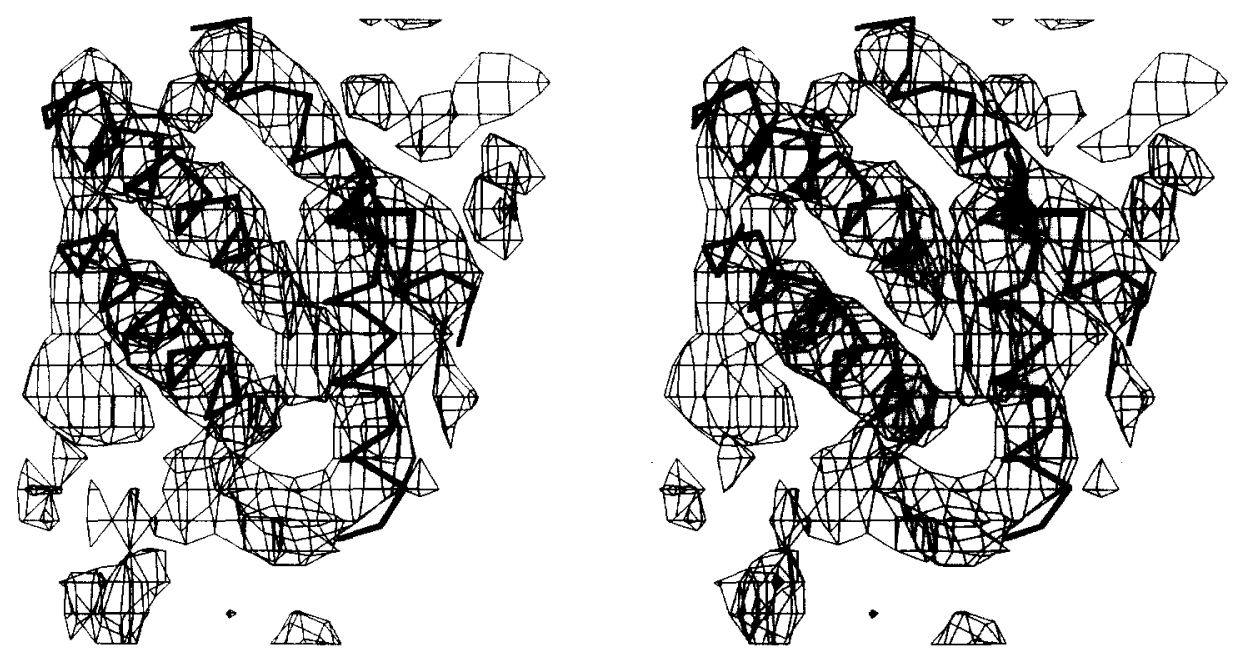

Figure 3. The $6 \AA$ resolution averaged electron density distribution. Density within a $25 \AA$ radius of the center of each molecule was averaged and contoured at $15 \%$ of the maximum (the r.m.s. value of the map). $C^{\alpha}$ models of 18 -residue $\alpha$-helices are included in the 4 major tubes of density to illustrate our interpretation. At a contour level of $30 \%$ of the maximum, only the 4 helices show electron density. 


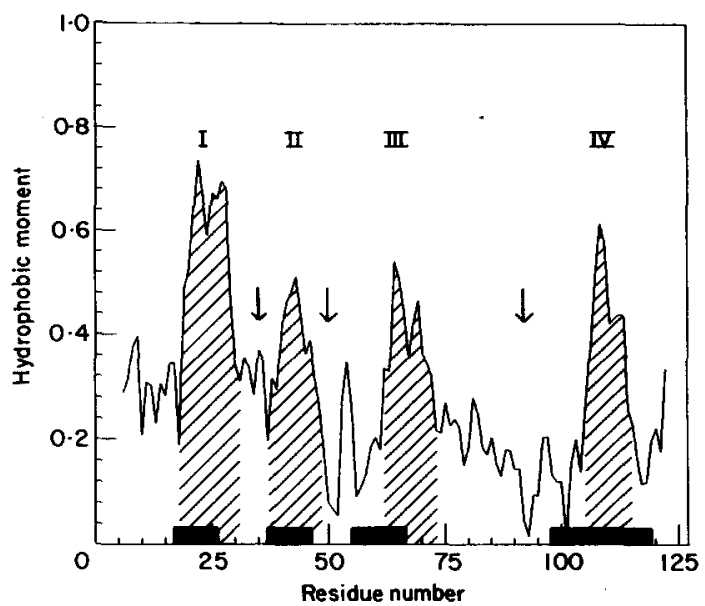

Figure 4. The 4 putative helix-forming regions of GM-CSF. Assuming $\alpha$-helical structure, the hydrophobic moment (Eisenberg et al., 1984) is calculated for all 11residue segments (residues $i-5$ through $i+5$ ) of the protein. Four stretches are located that have sequences characteristic of amphipathic helices in globular proteins (hatched areas labeled, I, II, III and IV). Bars on the $x$-axis show the 4 stretches of sequence that did not tolerate tripeptide deletions (Shanafelt \& Kastelein, 1989; numbering adjusted to match the human GM-CSF sequence by adding 3 to murine residue numbers beyond 30). Arrows (after residues 36, 50 and 92) mark the positions of introns in the GM-CSF gene (Miyatake et al., 1985).

between 4 and $3 \AA$ resolution, the non-crystallographic symmetry correlation is less than $0 \cdot 1$. The poor quality of the high-resolution phases appears to be due to the low heavy-atom occupancies and non-isomorphism. It is interesting to note that five out of eight unique heavy-atom binding sites fall at the interface between the two non-crystallographic symmetry-related molecules and would be expected to cause some changes in the packing. Also, the lack of stability of the crystals in storage buffer and the rapid decay of the high-resolution data support the idea that the crystal packing is somewhat fragile.

The four-helix structure we observed for GM-CSF correlates well with hydrophobic moment calculations based on its amino acid sequence (Eisenberg et al., 1984). These calculations identify exactly four segments that could form strongly amphipathic helices (Fig. 4). Further evidence to help to locate which parts of the sequence form the four helices comes from the results of scanning deletion analysis performed by Shanafelt \& Kastelein (1989). By deleting many different tripeptides from the structure, they showed that murine GM-CSF had four critical stretches that would not tolerate the deletions (see Fig. 4). The correspondence of these regions with those identified as potential amphipathic helices by the hydrophobic moment analysis suggests that these four segments do indeed form a structural core that is essential for activity. It is of interest to note that the intron positions in the GM-CSF gene fall between the purported helices (Fig. 4).
What about the structure of the remainder of the protein? The amino-terminal 15 residues and the carboxyl-terminal nine residues have been shown by deletion analysis to be expendable, and thus probably are not major contributors to the structural integrity of GM-CSF. The segments connecting putative helices I to II, and II to III (Fig. 4) are of appropriate length to make turns between the helices, and are probably too short to form any other significant secondary structure. The 30 residue segment between the putative helices III and IV is long enough to form some regular secondary structures and its conformation remains unknown. The major uninterpreted electron density (in the lower left region of Fig. 3) is probably due to this part of the chain filling in the more open edge of the core structure.

The structural analysis of GM-CSF provides a framework that should allow better planning of mutagenesis studies aimed at identifying residues important for interaction with the receptor. This four-helix domain structure appears to represent a novel protein fold, as other known four-helix bundles have helices that pack in a more parallel arrangement with angles near $20^{\circ}$ (Richardson, 1981). We are pursuing three approaches for obtaining the high-resolution structure: first, to carry out stepwise phase extension from the accurate low-resolution data; second, to continue the search for more and better derivatives, including the use of Se-Met labeled protein, which should skirt the problem of non-isomorphism (residues $36,46,79$ and 80 are Met); and third, to build and refine models for all possible matches of the critical sequences with the four observed helices, hoping that one will converge to the correct solution.

K.D. thanks the Alexander von Humboldt Foundation (Bonn, F.R.G.) for financial support. This work was supported by the Cornell Biotechnology Program, which is sponsored by New York State Science and Technology Foundation, a consortium of industries, the U.S. Army Research Office and the National Science Foundation. The Hewlett-Packard workstations used for molecular graphics were generously donated by Hewlett-Packard Corporation.

\section{References}

Antman, K. S., Griffin, J. D., Elias, M., Socinski, M. A., Ryan, L., Cannistra, S. A., Oette, D., Whitley, M., Frei, E., III \& Schnipper, L. E. (1988). Effect of recombinant human granulocyte-macrophage colony-stimulating factor on chemotherapy-induced myelosuppression. $N$. Engl. J. Med. 319, 593.

Boone, T., Crandall, L., Tsai, K. \& Zsabo, K. (1987). Purification of recombinant human granulocytemacrophage colony stimulating factor from $E$. coli J. Cell. Biochem. 11c, 176.

Brandt, S. J., Peters, W. P., Atwater, S. K., Kurtzberg, J., Borowitz, M. J., Jones, R. B., Shpall, E. J., Bast, R. C., Gilbert, C. J. \& Oette, D. H. (1988). Effect of recombinant human granulocyte-macrophage colony-stimulating factor on hematopoietic reconsti- 
tution after high-dose chemotherapy and autologous bone marrow translation. N. Engl. J. Med. 318, 869-876.

Brünger, A., Kuriyan, J. \& Karplus, M. (1987). Crystallographic R-factor refinement by molecular dynamics. Science, 235, 458-460.

Burgess, A. W., Begley, C. G.. Johnson, G. R., Lopez, A. F., Williamson, D. J., Mermod, J. J., Simpson, R. J., Schmitz, A. \& Delamarter, J. F. (1987) Purification and properties of bacterially synthesized human granulocyte-macrophage colony stimulating factor. Blood, 69, 43-5l.

Cebon, J., Nicola, N., Ward, M., Gardner, I., Dempsey, P.. Layton, J., Dührsen, U., Burgess. A. W., Nice, E. \& Morstyn, G. (1990). Granulocyte-macrophage colony stimulating factor from human lymphocytes. J. Biol. Chem. 265, 4483-4491

Chiba, S., Shibuya, K., Miyazono, K., Tojo, A., Oka, Y., Miyagawa, K. \& Takaku, F. (1990). Affinity purification of human granulocyte macrophage colonystimulating factor receptor $\alpha$-chain. J. Biol. Chem. 265, 19777-19781

Chothia, C., Levitt. M. \& Richardson, D. (1981). Helix to helix packing in proteins. J. Mol. Biol. 145, 215-250.

Clark, S. C. \& Kamen, R. (1987). The human hematopoietic colony-stimulating factors. Science, 236 . $1229-1237$.

Dickerson, R. E., Weinzierl, J. E. \& Palmer. R. A. (1968) A least-squares refinement method for isomorphous replacement Acta Crystallogr. sect. B, 24, 997-1003.

Dipersio, J., Billing, P., Kaufman, S., Eghtesady, P., Williams, R. E. \& Gasson. J. C. (1988) Characterization of the human granulocytemacrophage colony-stimulating factor receptor. J. Biol. Chem. 263, 1834-1841.

Eisenberg, D., Schwarz, E., Komaromy, M. \& Wall, R. (1984). Analysis of membrane and surface protein sequences with the hydrophobic moment plot. J. Mol. Biol. 179, 125-142.

Groopman, J. E.. Mitsuyasu, R. T., DeLeo, M. J., Oette, D. H. \& Golde, D. W. (1987). Effect of recombinant human granulocyte macrophage colony-stimulating factor on myelopoiesis in the acquired immunodeficiency syndrome. $N$. Engl. J. Med. 317, 593-598.

Grossberg. H. S. Bonnem, E. M. \& Buhles, W. C.. Jr (1989). GM-CSF with ganciclovir for the treatment of CMV retinitis in AIDS. N. Engl. J. Med. 320, 1560.

Hamlin, R. C. (1985). Multiwire area $\mathrm{X}$-ray diffractometers. Methods Enzymol. 114, 416-451.

Howard, A. J.. Nielsen, C. \& Xuong, Ng. H. (1985). Software for a diffractometer with multiwire area detector. Methods Enzymol. 114, 452-472.
Kaushansky, K.. O'Hara, P. J., Hart, C. E.. Forstrom, J. W. \& Hagen, F. S. (1987). Role of carbohydrate in the function of human granulocyte-macrophage colony-stimulating factor. Biochemistry, 26 $4861-4867$.

LaLonde. J. M.. Harna. L. S.. Rattoballi, R.. Berman H. M. \& Voet. D. (1989). Crystallization and preliminary X-ray studies of recombinant human granulocyte-macrophage colony stimulating factor. $J$. Mol. Biol. 205. 783-785.

Metcalf, D. (1989). The molecular control of cell division. differentiation commitment and maturation in hematopoietic cells. Nature (London), 339, 27-30).

Miyatake, S., Otsuka, T. Yokota, T.. Lee. F. \& Arai. K (1985). Structure of the chromosomal gene for granulocyte-macrophage colony stimulating factor: comparison of the mouse and human genes. $E M B O J .4$ $2561-2568$.

Reichert, P. Cook, W. J., Ealick, S. E.. Hammond, ('. S. Le, H. V.. Nagabhusan. T. L.. Trotta, P. P. \& Bugg C. E. $(1990)$. Crystallization and preliminary X-ray investigation of recombinant human granulocyte macrophage colony-stimulating factor. J. Biol. Chem. 265, 452-453.

Richardson. J. (1981). The anatomy and taxonomy of protein structure. Advan. Protein Chem. 34, 167-339

Rossmann. M. (A. \& Blow. D. M. (1962). The detection of sub-units within the crystallographic: asymmetric unit. Acta Crystallogr. 15, 24-31.

Schrimsher. .J. L.. Rose. K., Simona, M. G.\& Wingfield, P. (1987). Characterization of human and mouse granulocyte-macrophage colony-stimulating factors derived from Escherichia coli. Biochem. J. 247. $195-199$.

Shanafelt. A. B. \& Kastelein. R. A. (1989). Identification of critical regions in mouse granulocyte-macrophage colony-stimulating factor by scanning-deletion analysis. Proc. Nat. Acad. Sci. U.S.A. 86. $4872-4876$.

Wang. B. C. (1985). Resolution of phase ambiguity in macromolecular crystallography. Methods Enzymol. $115,90+112$

Wingfield. P.. Graber, P.. Moonen. P. Craig. S. \& Pain. R. H. (1988). The conformation and stability of recombinant-derived granulocyte-macrophage colony stimulating factors. Eur. J. Biochem. 173, 65-72.

Xuong, Ng. H.. Nielsen, C.. Hamlin, R. C. \& Anderson, 1). (1985). Strategy for data collection from protein crystals using a multiwire counter area detector diffractometer. J. Appl. Crystallogr. 18, 342-350. 